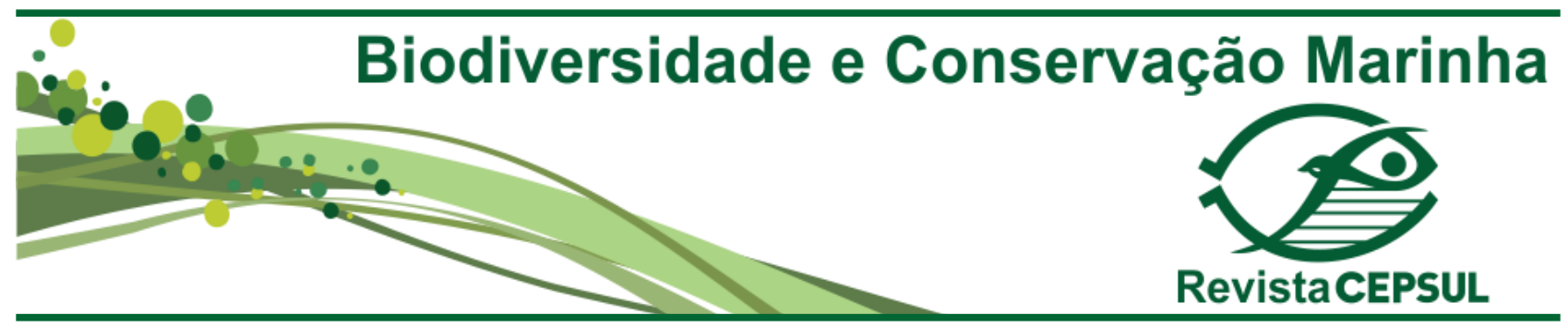

\title{
Fauna de cnidários do Ecossistema Babitonga: uma revisão crítica
}

\author{
Ana CARoline CABral $^{1} \&$ Miodeli Nogueira-JÚNior ${ }^{2}$
}

\begin{abstract}
${ }^{1}$ Programa de Pós-Graduação em Sistemas Costeiros e Oceânicos, Universidade Federal do Paraná - PGSISCO/UFPR, Rua Rio Grande do Norte, s/no, Mirassol CEP - 83.255-Ooo, Pontal do Paraná,PR, Brasil, ana.ccabral2@gmail.com;

${ }_{2}^{2}$ Departamento de Sistemática e Ecologia, Universidade Federal da Paraíba - UFPB, Cidade Universitária, CEP - 58051-90o , João Pessoa, PB,Brasil,miodeli@gmail.com.
\end{abstract}

Submetido em: 13/o3/2017; Aceito em: 27/o3/2018; Publicado em: 21/o1/2019

Resumo. Foi realizada uma ampla revisão da literatura sobre a cnidofauna do Ecossistema Babitonga (EB). Os trabalhos na região são poucos e recentes ( $95 \%$ realizados na última década), com o total de vinte e dois estudos, incluindo literatura cinza (11) e publicações em revistas científicas indexadas (11), sendo a região estuarina mais estudada que a plataforma. São registradas 113 espécies (18 Anthozoaria, 95 Medusozoa), dentre as quais oito espécies são exóticas. Não há informações sobre a influência da cnidofauna na economia e comunidade marinha do EB e trabalhos de perspectiva biológica e ecológica do grupo servirão de base para suprir essas lacunas. Sugere-se também monitoramento da biodiversidade local considerando que o EB está susceptível a introduções de espécies exóticas devido às atividades portuárias e maricultura, bem como proximidade com regiões já invadidas por espécies com elevado potencial invasor e de modificação da comunidade nativa.

Palavras-chave: Cnidaria, medusas, pólipos, síntese de conhecimento, estuários, Brasil.

\begin{abstract}
Cnidarian fauna from Babitonga ecosystem: a critical review. A comprehensive literature review regarding cnidarians from Babitonga Ecosystem (BE) is provided. Local studies are few, 11 grey literature and 11 articles from indexed scientific journals, recent ( $95 \%$ performed in the last decade), and mostly made on the estuarine area. A total of 113 species were recorded (18 Anthozoaria, 95 Medusozoa), eight of which are exotic. There is no information on cnidarian impacts on BE aquatic communities or local economy. Yet, local biological and ecological studies are lacking and thus are necessary. We also suggest the monitoring of local biodiversity, particularly considering that $\mathrm{BE}$ is vulnerable to the introduction of exotic species due to the high levels of local port and mariculture activities along with the proximity of ecosystems already invaded by aggressive species .
\end{abstract}

Keywords: Cnidaria, medusa, polyp, synthesis of knowledge, estuaries, Brazil. 


\section{Introdução}

O filo Cnidaria é um grupo muito diverso de invertebrados aquáticos, representado por águas vivas, anêmonas do mar, corais, hidrozoários, entre outros. Esses organismos possuem como principal característica a capacidade de produzir cnidócitos, células que sintetizam cápsulas com substância urticante (cnidas), utilizadas para captura de alimento e defesa do animal. Outra característica do grupo é a presença de dois padrões morfológicos, o pólipo e a medusa (geralmente séssil e livre natante, respectivamente). A ausência ou presença da fase medusóide no ciclo de vida divide os cnidários entre os subfilos Anthozoaria (anêmonas do mar, corais e zoantídeos) e Medusozoa (sifonóforos, medusas e hidróides), respectivamente (Daly et al., 2007; Haddad \& Marques, 2009).

A alternância de gerações é uma condição primitiva do subfilo Medusozoa, em que tipicamente há a fase polipóide assexuada e a fase medusóide sexuada, ainda assim algumas linhagens suprimiram parcial ou totalmente uma dessas fases (Cornelius, 1992), bem como associaram ambas as fases ocorrendo simultaneamente em uma só colônia (sifonóforos). Dessa forma, algumas espécies completam seu ciclo de vida somente no bentos ou no plâncton (holoplanctônicas) enquanto outras transitam entre ambos os compartimentos (meroplanctônicas), o que implica na riqueza e diversidade do grupo em determinados ecossistemas (Gibbons et al., 2010). A ocorrência desses organismos no plâncton e bentos os torna também agentes importantes na transferência de energia entre esses dois compartimentos, sendo atuantes na ciclagem de nutrientes (Bouillon et al., 2004).

Estudos sobre cnidários na costa brasileira vêm aumentando nos últimos anos, no entanto sua riqueza e diversidade são ainda insuficientemente conhecidas, principalmente devido à subamostragem da maioria das áreas costeiras e oceânicas do país (Haddad \& Marques, 2009). Tais informações são básicas para a realização de pesquisas relacionadas à biologia, ecologia e mesmo filogenia desses organis- mos (Marques et al., 2003), bem como sua relação com o ecossistema onde está inserido. Ecossistemas estuarinos comumente estão sob grande pressão antrópica (e.g. Cremer et al., 2006), destacando-se, entre outros aspectos, as espécies exóticas as quais tem sido dada uma grande atenção recente (e.g. Molnar et al., 2008; Gravili, 2017) e são consideradas uma ameaça aos ecossistemas marinhos. Entre os diversos grupos taxonômicos, os cnidários são particularmente importantes com diversas espécies exóticas, muitas das quais causando impactos consideráveis nos ecossistemas invadidos (Creed et al., 2017). Neste contexto, o presente estudo traz uma ampla revisão da literatura sobre Cnidaria da Baía da Babitonga e plataforma continental adjacente, conhecido como Ecossistema Babitonga (Gerhardinger et al., 2017) com o objetivo de dar subsídios a um diagnóstico ambiental amplo visando melhoria da gestão dos recursos naturais desse importante ecossistema (ver Gerhardinger et al., 2018 - editorial do Especial Babitonga).

\section{Metodologia}

Uma revisão crítica do conhecimento de Cnidaria do Ecossistema Babitonga (EB) foi realizada com base em literatura cinza (resumos de congressos, monografias, dissertações e teses) e trabalhos publicados em periódicos científicos indexados nacionais e internacionais até o ano de 2016. Foi utilizada a experiência prévia dos autores em estudos na região (e.g. Nogueira-Júnior \& Souza-Conceição, 2010; Nogueira-Júnior, 2012; Cabral, 2013; Nogueira-Júnior et al., 2015; Nogueira-Júnior \& Oliveira, 2017), e buscas na internet usando o Google acadêmico e a Plataforma Lattes (http://lattes.cnpq.br/) para a obtenção dos trabalhos. As buscas foram realizadas até fevereiro de 2017. Combinações entre as palavras chave "Babitonga", "Cnidaria", "São" Francisco do Sul”, "Itapoá", "Barra do Sul" e "Parque Estadual Acaraí” foram utilizadas nas buscas. Todos os trabalhos encontrados (publicações em periódicos científicos, capítulos de livros e literatura cinza) foram incluídos na presente revisão, sendo que para a lista de espécies, a litera- 
tura cinza foi substituída pela respectiva publicação quando disponível. Foram considerados também dados não publicados e observações pessoais dos autores. O sistema de classificação das espécies seguiu a indicada na WorRMS (marinespecies.org; até fevereiro de 2017).

\section{Resultados e discussão}

Os estudos com cnidários no EB são bastante recentes (Yoshimura, 1997) e esporá- dicos, com apenas onze artigos em revistas científicas indexadas e onze trabalhos de literatura cinza (Figura 1A). A grande maioria (95\%) dos estudos concentra-se na última década, $\mathrm{e}$ $78 \%$ (88 spp.) dos cnidários atualmente encontrados no EB foram registradas somente a partir de 2011 (Figura 1B). Medusozoa foi o grupo mais abordado com 17 estudos (77\%), comparado com apenas sete que incluem Anthozoaria (32\%). Entre os estudos de Medusozoa, a grande maioria (12) refere-se aos estágios planctô-
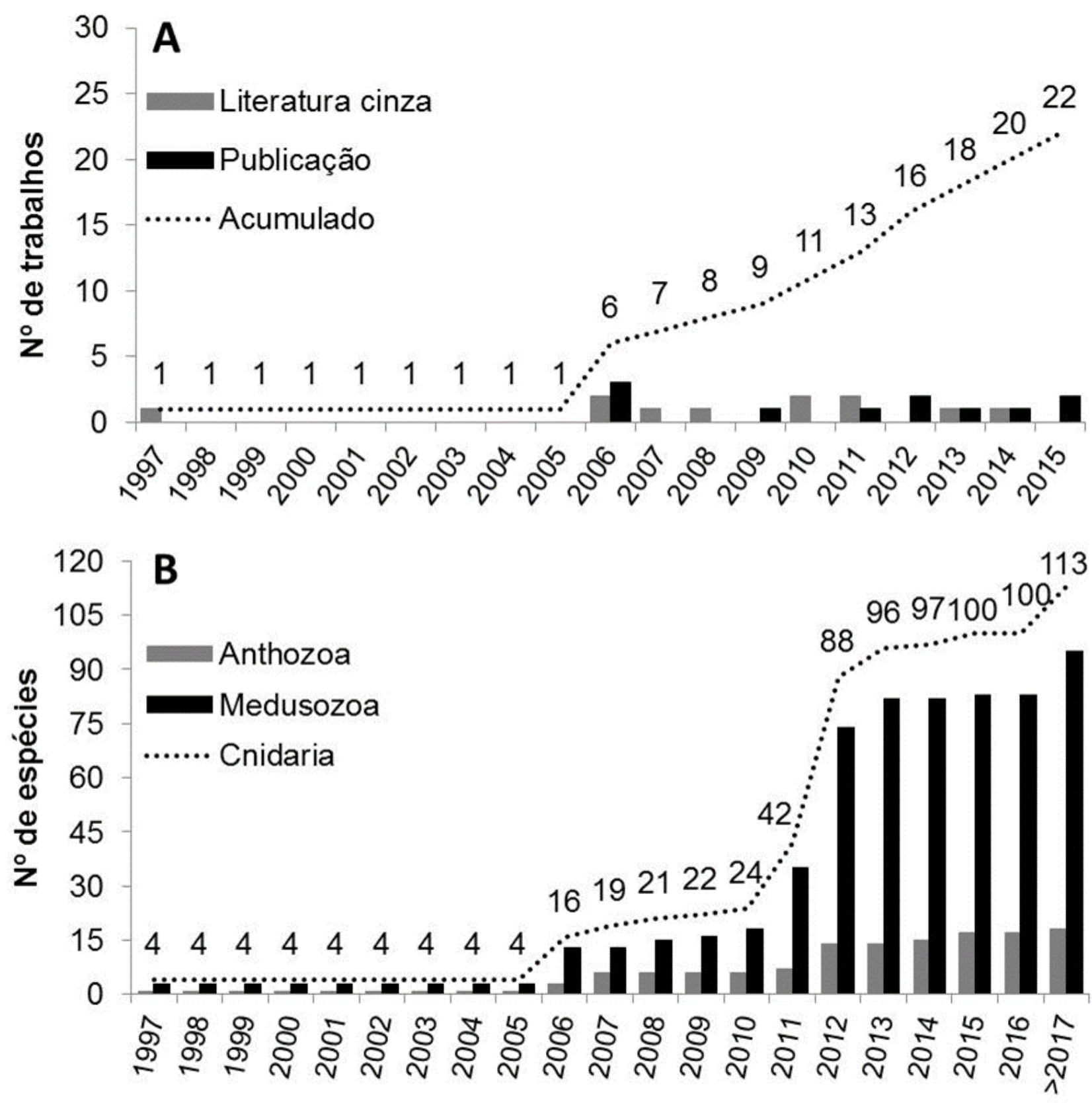

Figura 1. Evolução do número de estudos com cnidários (A) e de espécies dos subfilos Anthozoaria e Medusozoa (B) registradas no Ecossistema Babitonga. 


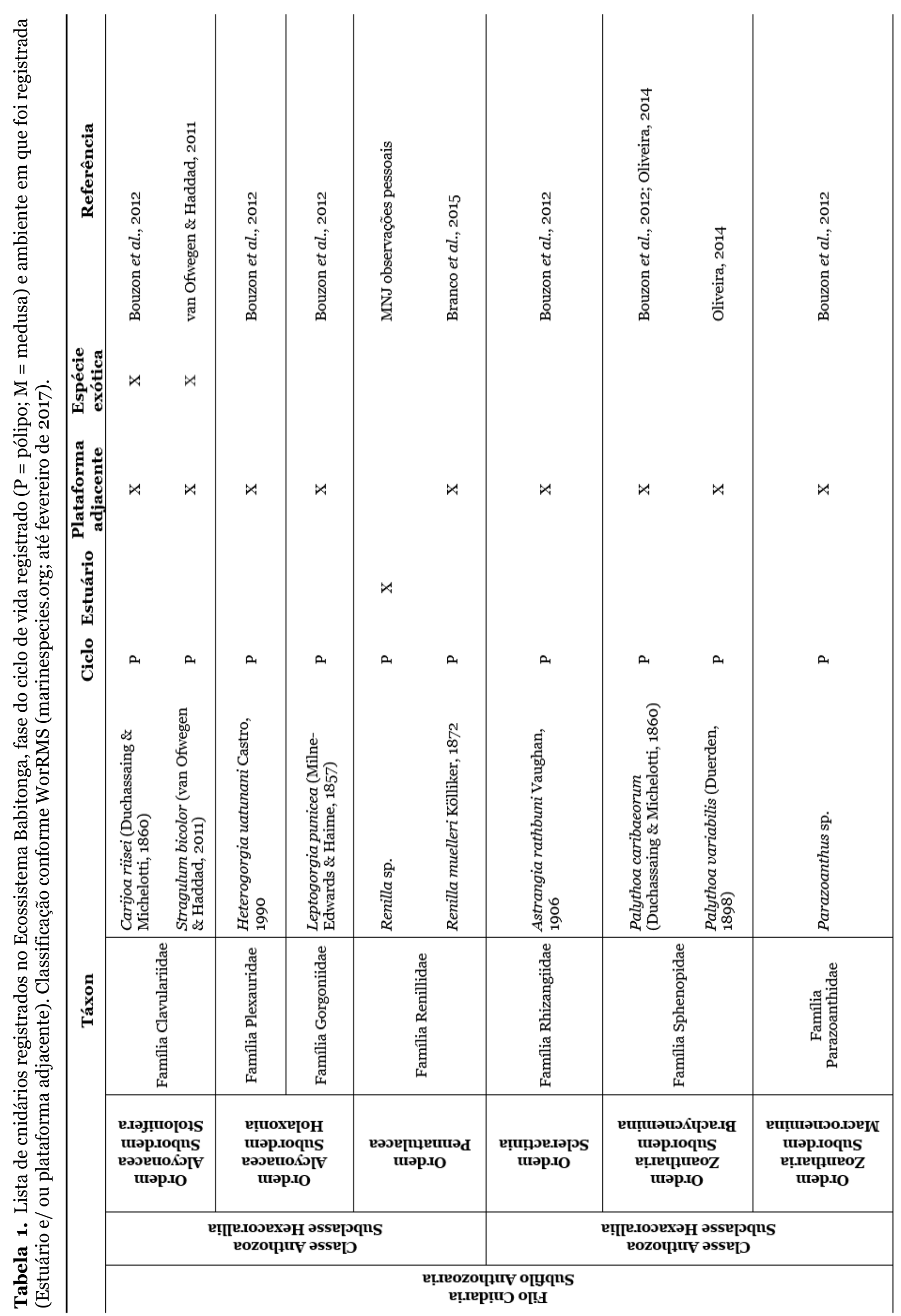

Revista CEPSUL - Biodiversidade e Conservação Marinha, 8: eb2019oo3 


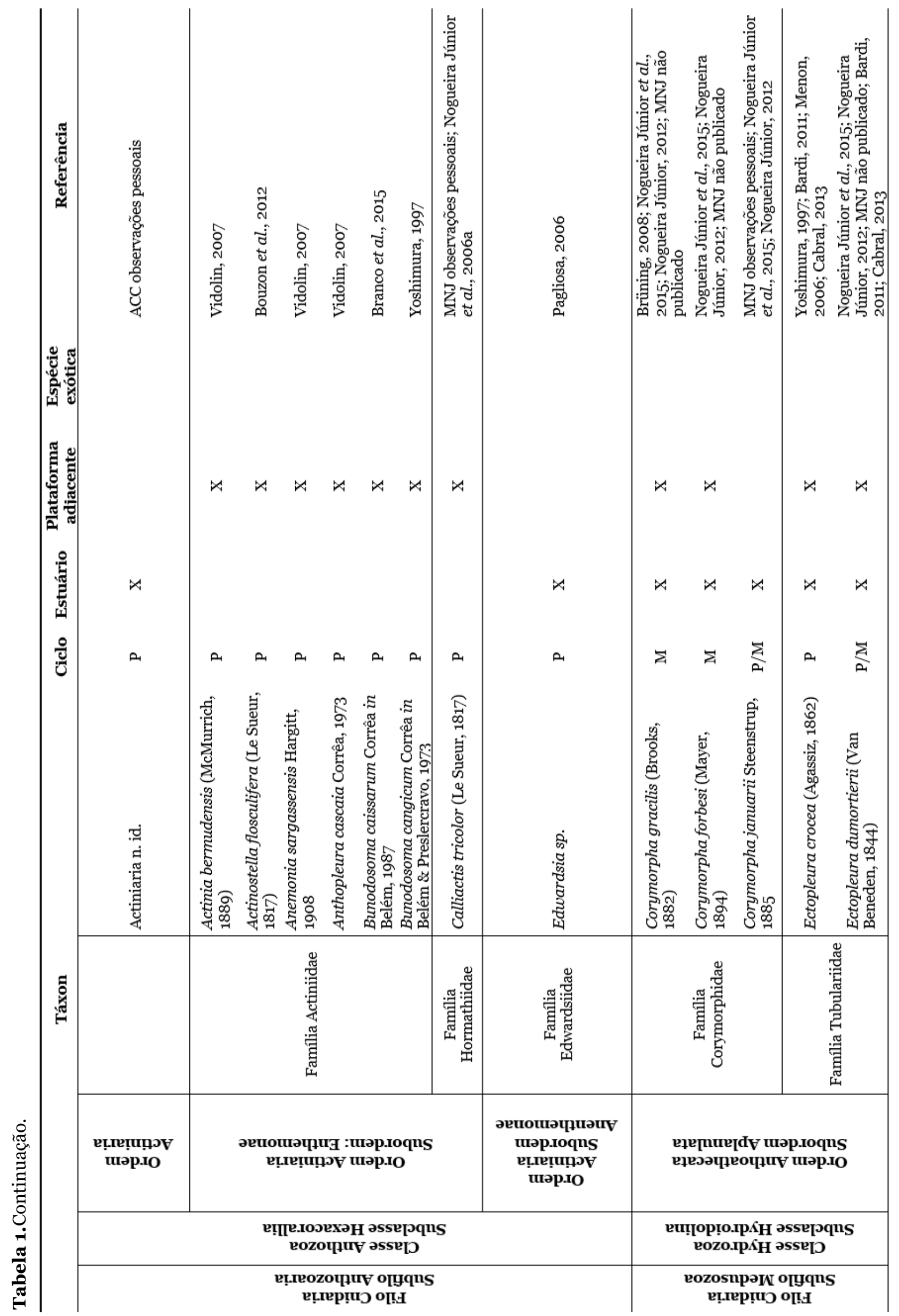




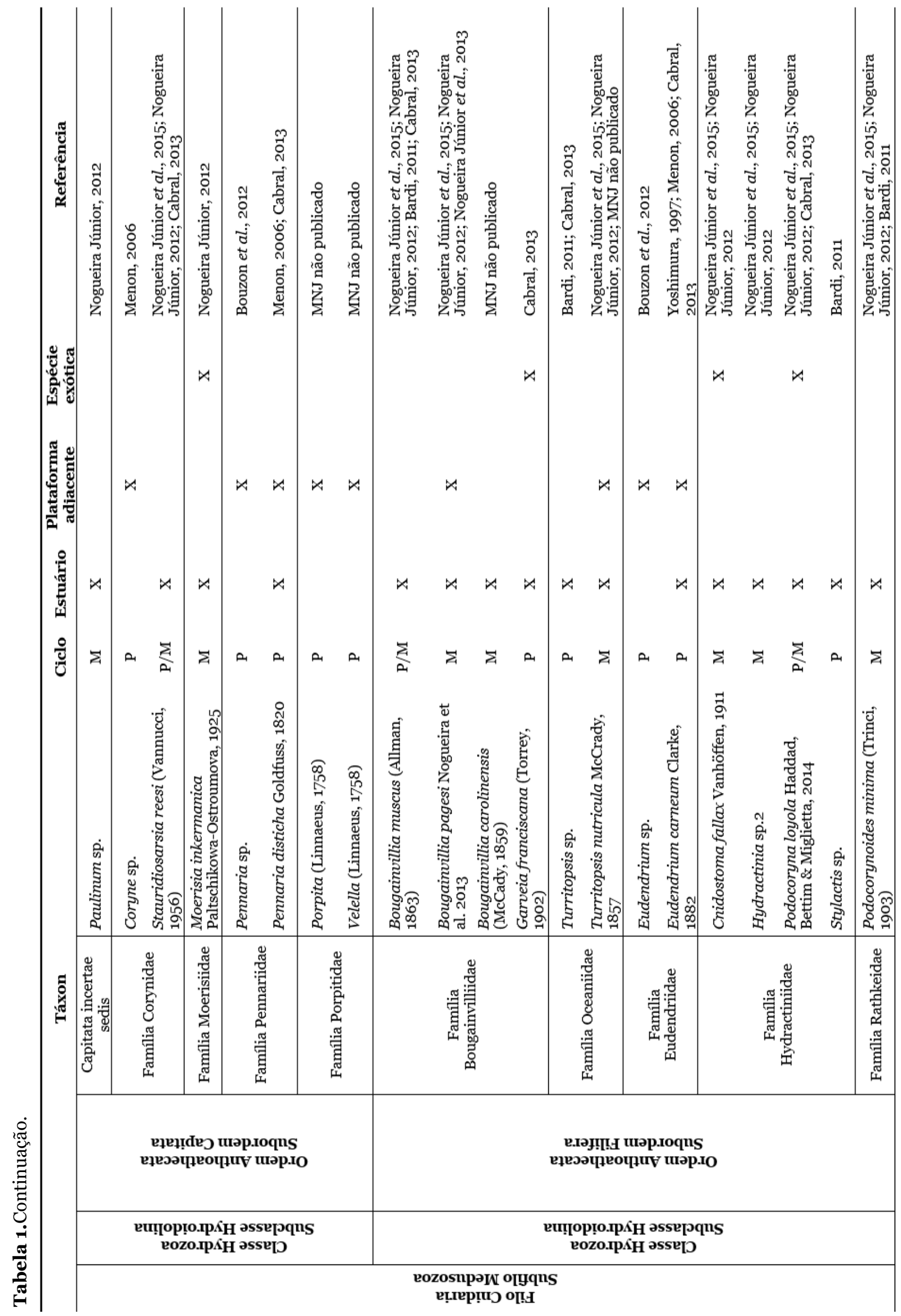

Revista CEPSUL - Biodiversidade e Conservação Marinha, 8: eb2019oo3 


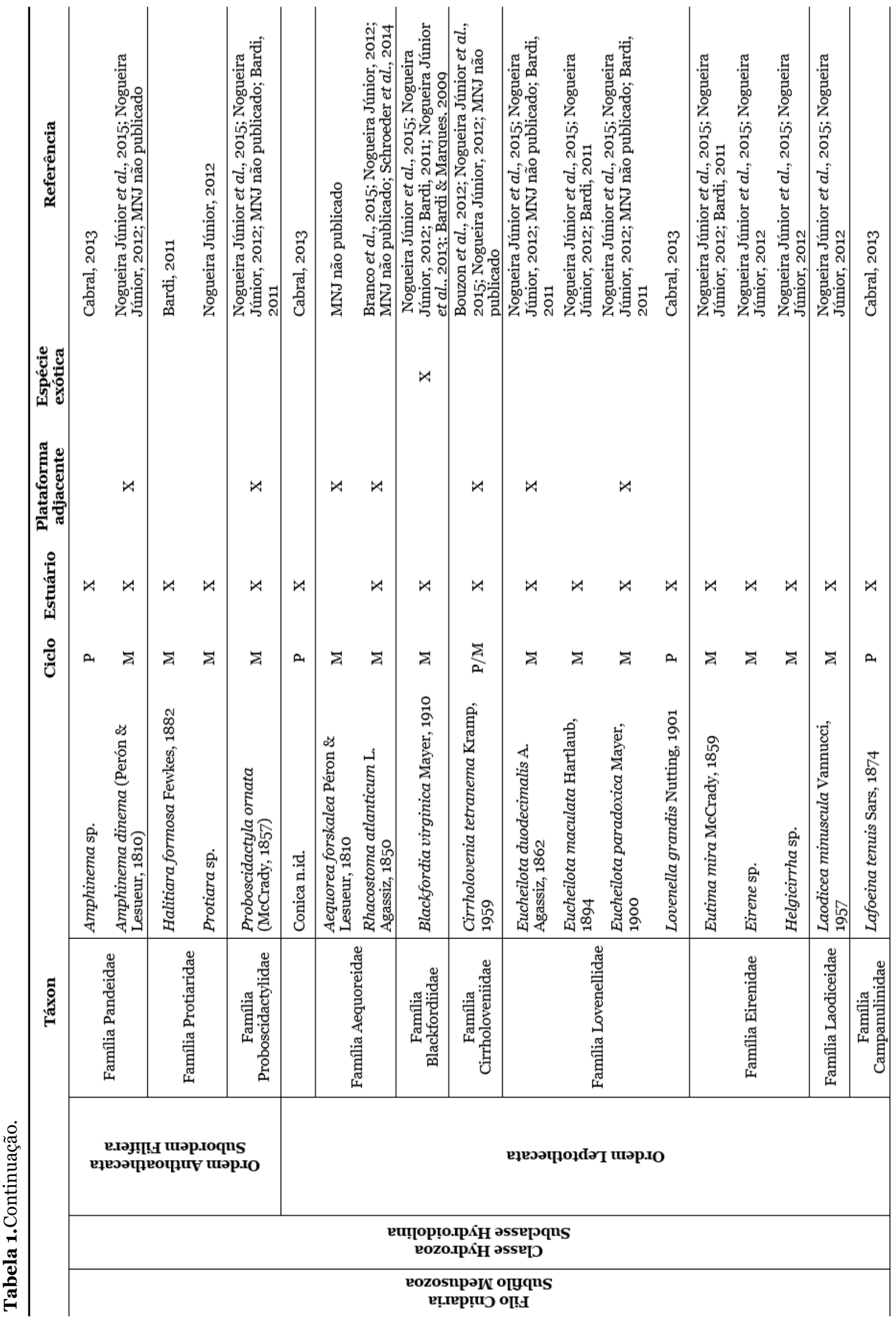




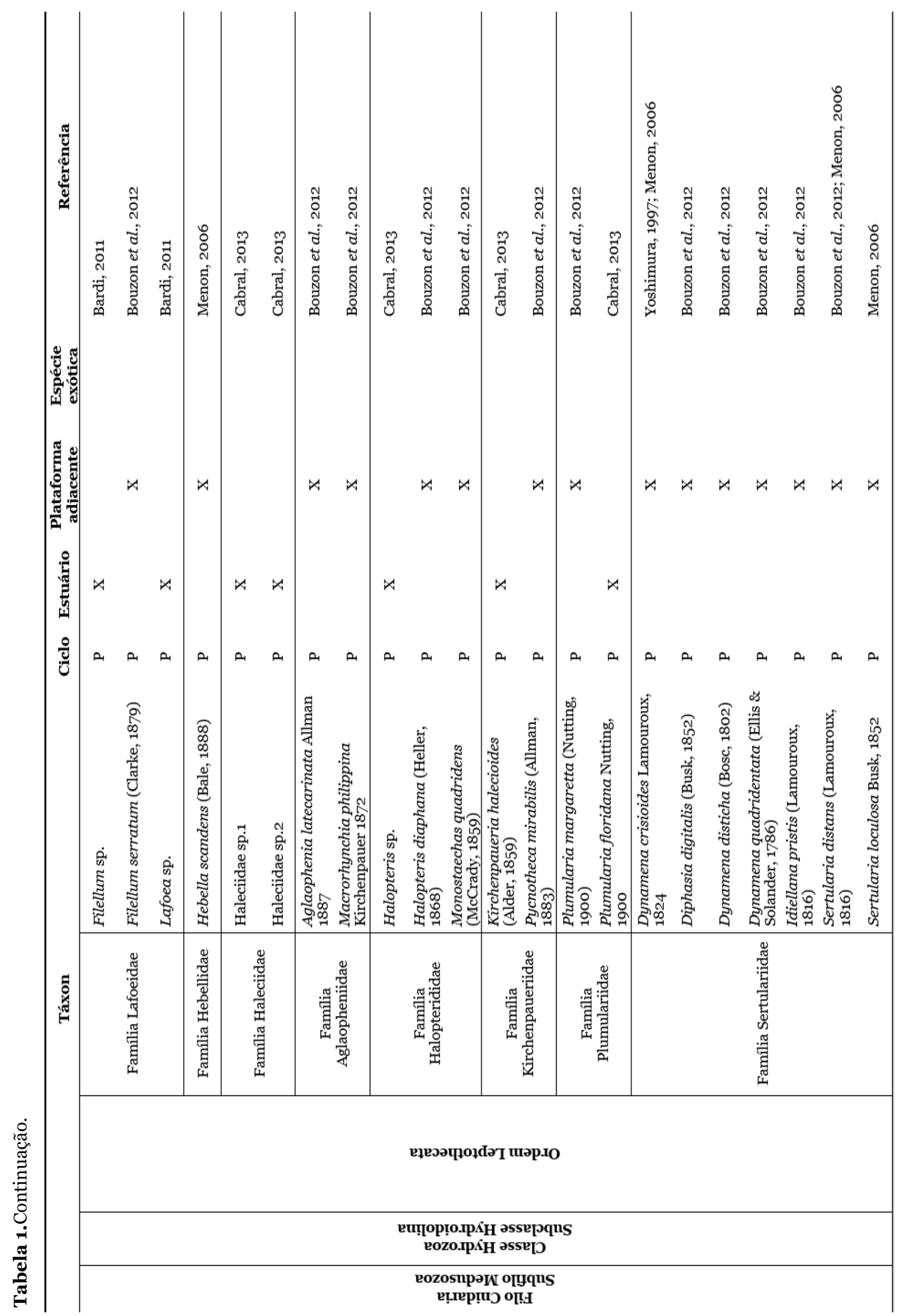

Revista CEPSUL - Biodiversidade e Conservação Marinha, 8: eb2019oo3 


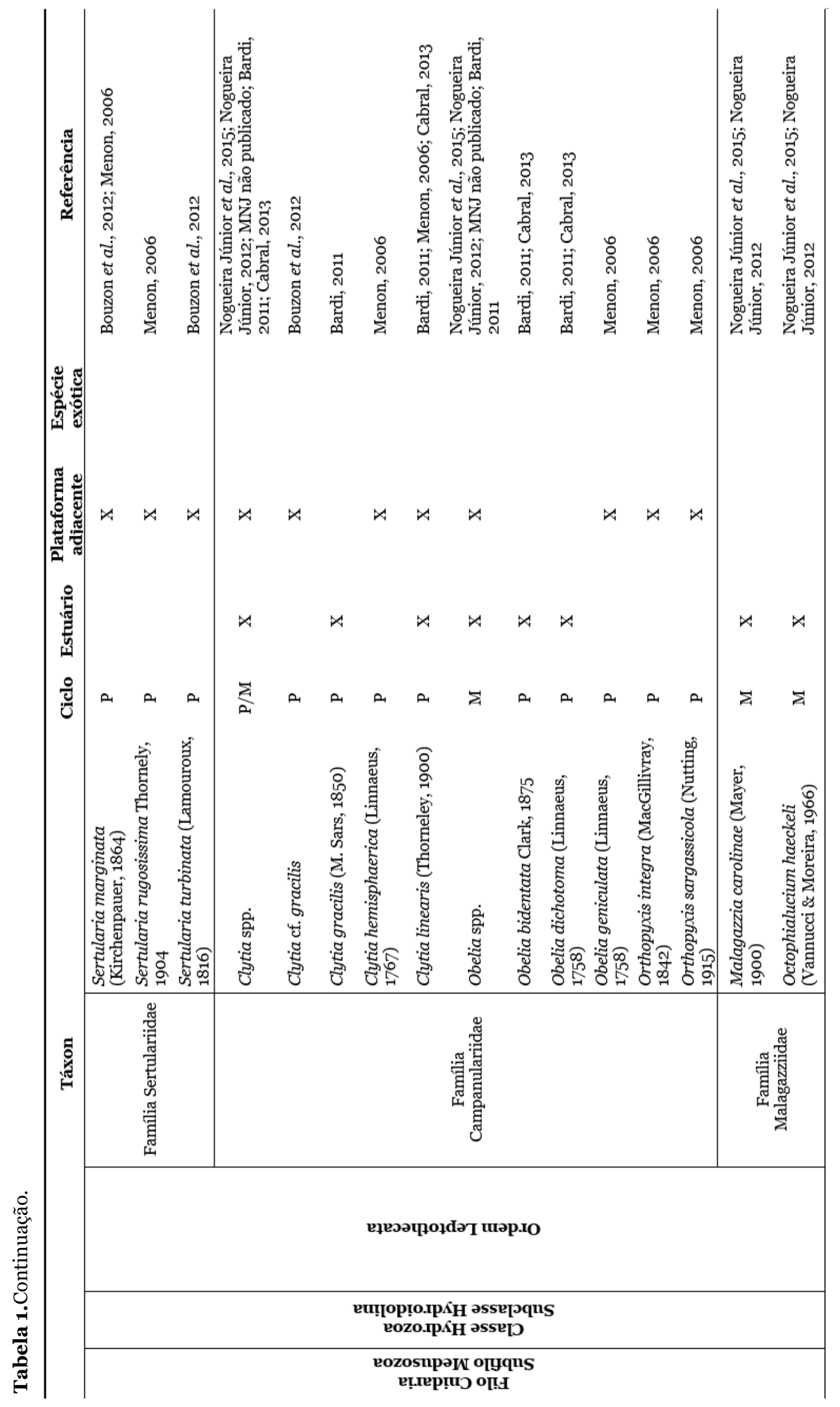

Revista CEPSUL - Biodiversidade e Conservação Marinha, 8: eb2019oo1 


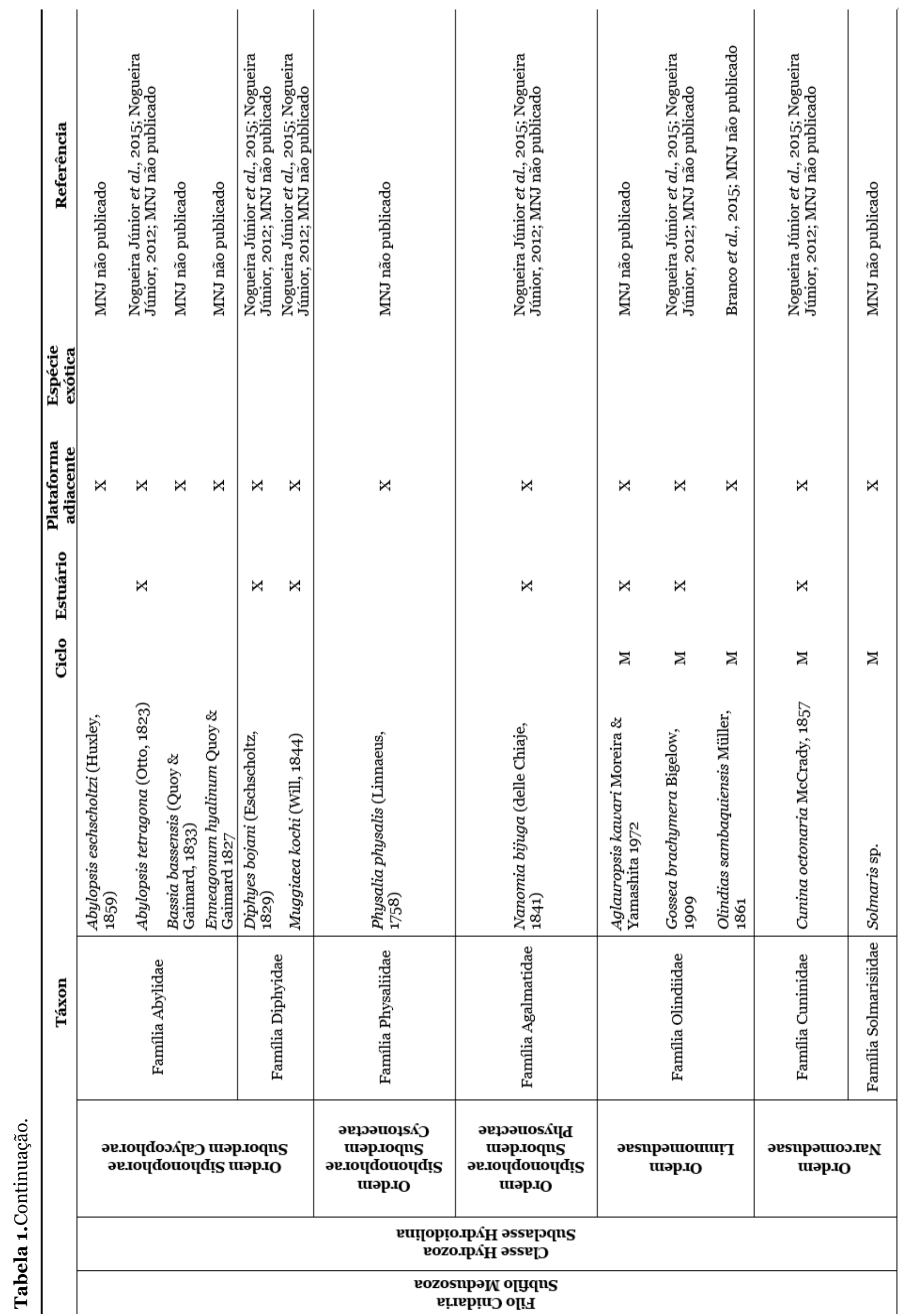

Revista CEPSUL - Biodiversidade e Conservação Marinha, 8: eb2019oo3 


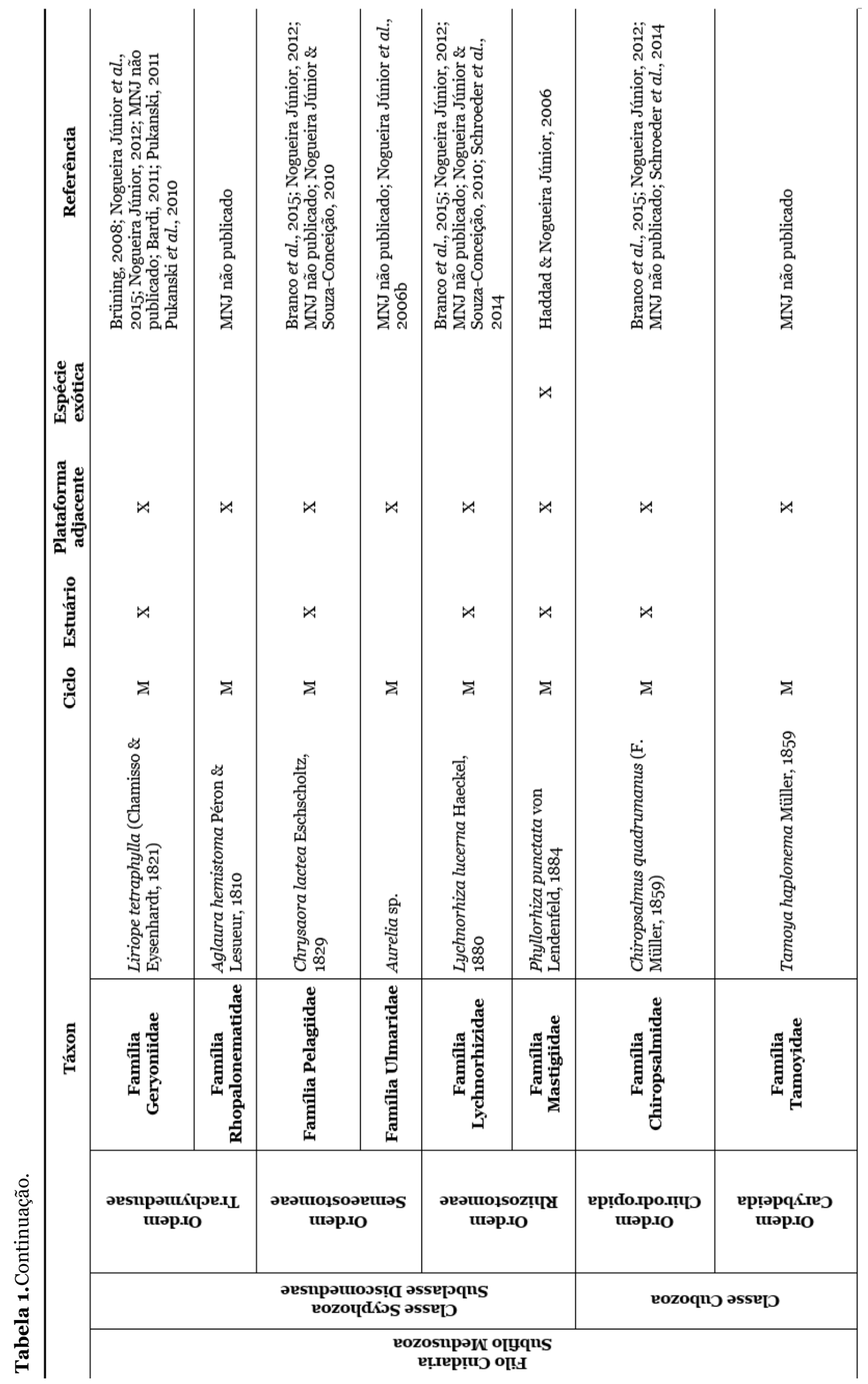

Revista CEPSUL - Biodiversidade e Conservação Marinha, 8: eb2019oo1 
nicos e apenas cinco à fase polipóide (Tabela 1).

A maioria dos trabalhos concentra-se em desenvolvimento e distribuição sazonal e/ ou espacial (Haddad \& Nogueira Júnior, 2006; Nogueira Júnior \& Souza-Conceição, 2010; Pukanski, 2011; Oliveira, 2014), seguido de trabalhos de inventariamento e/ou cunho taxonômico (Brünning, 2008; Bardi, 2011; Bouzon et al., 2012; Nogueira Júnior, 2012; Cabral, 2013) incluindo descrições de novas espécies tanto de Anthozoaria (van Ofwegen \& Haddad, 2011) quanto de Medusozoa (Nogueira Júnior et al., 2013). Outros temas estudados na região incluem o papel das medusas como fauna acompanhante na pesca (Schroeder et al., 2014; Branco et al., 2015) e associações de simbiose (Nogueira Júnior et al., 2006a).

Foram registrados 123 morfotipos de cnidários e 113 espécies, visto que as 10 que continham a identificação "sp.", "spp." ou "cf." e congênere registrado, não foram contabilizados na riqueza, a fim de evitar superestimação de espécies. Dentre as 113 espécies, 18 pertencem ao subfilo Anthozoaria e 95 ao Medusozoa. A predominância de Medusozoa pode ser reflexo do maior esforço amostral nesse grupo, já que Anthozoaria tem mais espécies descritas que Medusozoa (Haddad \& Marques, 2009). Ainda assim é comum maior riqueza local deste último (Bouzon et al, 2012; Bumbeer \& Rocha, 2012; Marques et al., 2013).
As 18 espécies de Anthozoaria estão distribuídas em cinco Octocorallia e 13 Hexacorallia, sendo a família Actiniidae a de maior riqueza (6 spp.). A grande maioria das espécies foram registradas na plataforma adjacente (16), em ilhas dos arquipélagos de Tamboretes e das Graças (Bouzon et al., 2012, Oliveira, 2014), incrustantes em costões rochosos de praias (Yoshimura, 1997; van Ofwegen \& Haddad, 2011), epibiontes em Brachyura (Nogueira Júnior et al., 2006a) ou como fauna acompanhante de arrastos de camarão (Branco et al., 2015). Edwardsia sp. foi a única representante de Anthozoaria na região estuarina, no entanto agregados de Actiniaria n. id., (A.C. Cabral, observações pessoais) e Renilla sp. (M. Nogueira Júnior, observações pessoais) já foram observados em substratos artificiais e em arrasto de fundo dentro do estuário, respectivamente. De fato, espera-se uma maior riqueza de Anthozoaria em áreas de maior salinidade e menor turbidez, visto que são organismos predominantemente marinhos e muitos são zooxantelados, como espécies de Palythoa e muitos escleratíneos (Haddad \& Marques, 2009). Ainda assim, a riqueza de antozoários no estuário certamente está subestimada devido à falta de estudos. De acordo com a figura 2, pode-se perceber a grande escassez de estudos com antozoários na região, restritos apenas à região de plataforma e áreas externas do estuário, existindo assim uma enorme lacuna. Também não existem in-

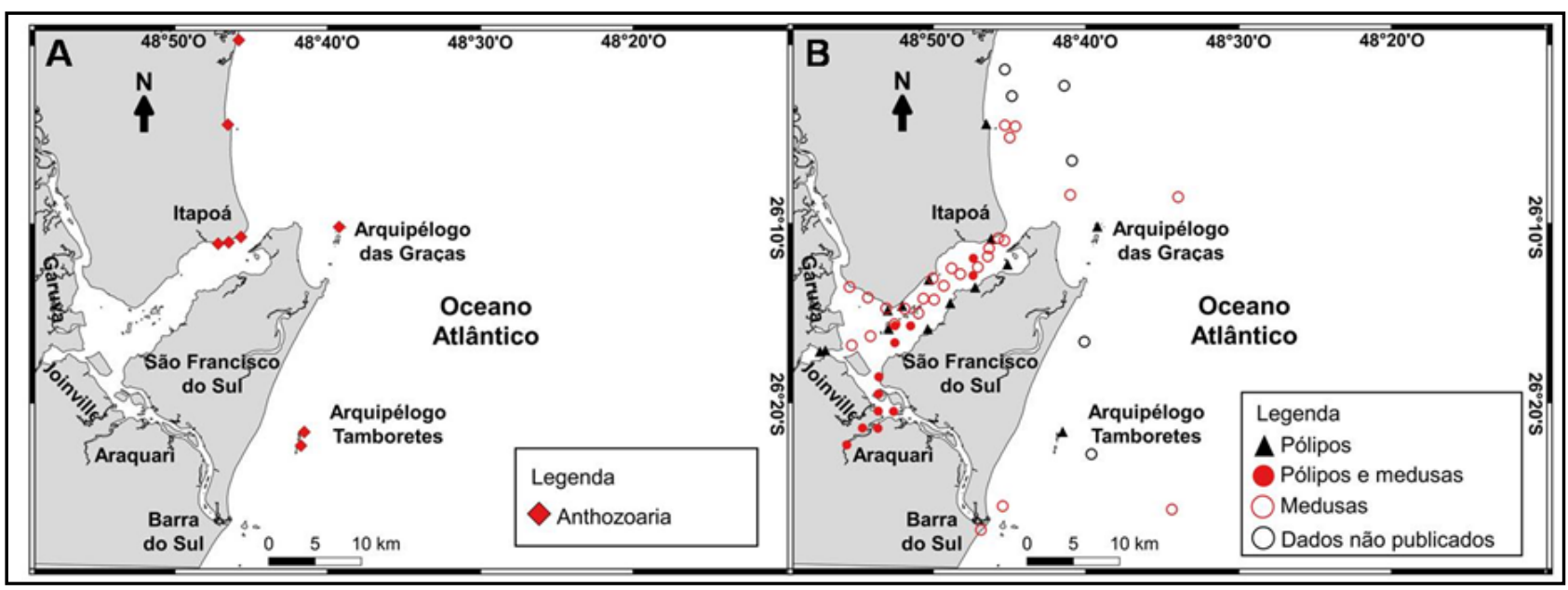

Figura 2. Distribuição dos pontos amostrados em estudos já realizados no Ecossistema Babitonga, separados entre Anthozoaria (A) e Medusozoa (B). Em Medusozoa os pontos amostrados em Branco et al. (2015) e Schroeder et al. (2014) não foram incluídos devido a indisponibilidade de coordenadas nos respectivos trabalhos. 
formações quantitativas para o grupo na região.

Dentre as 95 espécies de Medusozoa registradas até o momento no EB, 89 (94\%) pertencem à classe Hydrozoa, particularmente das ordens Leptothecata (47 spp.) e Anthoathecata (27 spp.). Outros grupos são pouco diversos na região como os sifonóforos (8 spp.), cifomedusas (4 spp.), limnomedusas (3 spp.), e narco, traqui e cubomedusas ( 2 spp. cada; Tabela 1). Esse padrão é o esperado para um ecossistema costeiro e/ou estuarino como a Babitonga. Em geral, os leptotecados e antoatecados incluem os estágios de pólipo e medusa no ciclo de vida e são particularmente diversificados em águas rasas (Gibbons et al., 2010). Outros cnidários meroplanctônicos como as limnomedusas, os cifozoários e cubozoários são pouco diversos, com menos de 50 espécies registradas em todo o Brasil somando todos esses grupos (Oliveira et al., 2016). Já os grupos holoplanctônicos como os sifonóforos, narco e traquimedusas, têm maior diversidade em águas oceânicas, sendo pobres em número de espécies em regiões costeiras (Gibbons et al., 2010), o que é particularmente verdadeiro no caso do EB que tem uma plataforma continental extensa com mais de $150 \mathrm{~km}$ (e.g. Nogueira Júnior \& Brandini, 2018).

Embora cnidários sejam animais predominantemente marinhos, o número de espécies de Medusozoa registrado dentro do estuário (66 spp.) e na plataforma (67 spp.) foi semelhante, o que parcialmente é reflexo da ausência de levantamentos extensos de Medusozoa na plataforma adjacente (Figura 2), com apenas oito estudos. Mesmo assim, diversas espécies, em geral meroplanctônicas, são exclusivas ou preferencialmente estuarinas e/ou de águas salobras, que toleram ampla variação de salinidade, contribuindo com a grande diversidade de cnidários no estuário, como Aglauropsis kawari, Blackfordia virginica, Amphinema spp., Clytia linearis, Bougainvillia muscus, $B$. carolinensis, Cnidostoma fallax, Corymorpha januarii, Eirene sp., Eucheilota maculata, Eutima mira, Garveia franciscana, Gossea brachymera, Helgicirrha sp., Malagazzia carolinae, Podocoryna loyola e Stauridiosaria reesi.
Na região estuarina houve também o primeiro registro do Hydrozoa Lovenella grandis para o Atlântico Sul e da fase polipóide Conica n.id., raramente observada em campo, e que pode corresponder a uma (ou mais) das espécies de medusas das famílias Lovenellidae e Eirenidae como Eucheilota maculata, E. paradoxica e Eutima mira, já registradas no local (Tabela 1) (Bouillon et al., 2004; Cabral, 2013).

Dados quantitativos para os Medusozoa na região são esparsos. Os hidróides mais abundantes são principalmente Ectopleura spp. no inverno e Clytia spp., Podocoryna loyola, Garveia franciscana e Obelia dichotoma no verão (Cabral, 2013). Entre as medusas, picos de abundância foram registrados na primavera, com densidades totais sempre $<120$ ind. $\mathrm{m}^{-3}$. As espécies tipicamente dominantes são Liriope tetraphylla, Obelia spp. e Clytia spp., além de Muggiaea kochi, Ectopleura dumortieri, Proboscidactyla ornata, Podocoryna loyola, Blackfordia virginica e Corymorpha gracilis, que também podem ser eventualmente abundantes (Nogueira Júnior et al., 2015). Entre as macromedusas, Chysaora lactea é a dominante dentro do estuário e a mesma e Lychnorhiza lucerna dominam a região de plataforma (Nogueira Júnior \& Souza-Conceição, 2010; Schroeder et al, 2014). Porém, não existem dados de sazonalidade disponíveis, exceto para Phyllorhiza punctata com ocorrência da fase medusoide entre o verão e início do outono (Haddad \& Nogueira Júnior, 2006).

Mundialmente, os cnidários são um dos grupos dominantes em termos de espécies marinhas invasoras, com grande possibilidade de riscos ambientais e econômicos (Molnar et al., 2008). Dentre os 113 cnidários registrados no $\mathrm{EB}$, dois $(\sim 2 \%)$ antozoários e seis $(\sim 5 \%)$ Medusozoa são possivelmente exóticos (Tabela 1). Carijoa rïsei é considerada historicamente introduzida em diversas regiões do mundo (Concepción et al., 2010), enquanto Stragulum bicolor é uma espécie recentemente descrita e considerada introduzida devido aos recentes registros (a partir do ano 2000) em áreas historicamente estudadas (van Ofwegen \& Haddad, 2011). Embora tenham sido encontradas somente na plataforma adjacente do EB ( $S$. 
bicolor em costões de Itapoá; $C$. rïsei em ilhas costeiras), são espécies tipicamente estuarinas e de áreas portuárias (Cangussu et al., 2010; Altvater \& Coutinho, 2015), sugerindo o tráfego marítimo como principal meio de introdução (Kahng \& Grigg, 2005). Ambas as espécies têm rápido recrutamento e desenvolvimento em substratos limpos e sobre a comunidade já estabelecida (Kahng \& Grigg, 2005; Cangussu et al., 2010; Altvater \& Coutinho, 2015). Essa característica, aliada ao transporte marítimo como principal meio de dispersão, reforça o potencial de introdução e estabelecimento dessas espécies em novas áreas, inclusive na região estuarina do EB. Não há evidências de impactos negativos de $S$. bicolor nas comunidades hospedeiras (Altvater \& Coutinho, 2015), ainda assim ressalta-se a importância do monitoramento dessas espécies devido seu potencial de recrutamento e sobreposição da comunidade.

Blackfordia virginica, Garveia franciscana, Moerisia inkermanica e Phyllorhiza punctata são espécies com um histórico de invasões bem conhecido em diversos locais do mundo incluindo o Brasil (Haddad \& Nogueira Júnior, 2006; Bardi \& Marques, 2009; Cangussu et al., 2010; Gravili, 2017). A hidromedusa Cnidostoma fallax recentemente tem aparecido em diversos estuários do sul e sudeste do Brasil incluindo a Babitonga (Nogueira Júnior, 2012), sendo provavelmente exótica, originária da costa oeste africana de onde era considerada endêmica (Nogueira Júnior \& Costa, 2019). Podocoryna loyola é provavelmente exótica no sul do Brasil. Apesar de estudos locais com hidróides desde os anos 1980, essa espécie foi encontrada apenas recentemente (desde 2007) incrustada em substratos artificiais, característica de espécies invasoras (Haddad et al., 2014). Garveia franciscana é considerada estabelecida no litoral do Paraná (Bumbeer \& Rocha, 2016). Apesar da difícil avaliação devido à escassez de estudos, $B$. virginica e $P$. loyola também provavelmente têm populações estabelecidas no EB, já que têm sido comumente encontradas em abundância em diferentes anos de amostragem e/ou diferentes estágios do ciclo de vida (Bardi, 2011; Nogueira Júnior, 2012; Cabral, 2013). A população de $P$. puncta- ta, ao contrário, aparentemente declinou, pelo menos o estágio medusoide que não tem sido observado na região desde 2005 (Haddad \& Nogueira Júnior, 2006). Não se tem informações suficientes para avaliar se $C$. fallax e $M$. inkermanica mantêm populações estabelecidas na região. Exceto G. franciscana, que não produz medusa livre, todas as outras espécies não nativas de medusozoários são meroplanctônicas, assim possíveis vias de introdução incluem tanto estágios livre-natantes, presentes em água de lastro, quanto estágios sésseis incrustados em cascos de embarcações, que são os principais meios de transporte de espécies marinhas, juntamente com a indústria da maricultura (Molnar et al., 2008).

Com exceção dos octocorais $C$. rïisei e $S$. bicolor, que ocorreram somente na plataforma, os medusozoários não nativos foram registrados dentro do estuário (Tabela 1). Maior número de espécies introduzidas é esperado para regiões estuarinas, visto que ambientes com atividades portuárias, intenso tráfego marítimo e atividades de maricultura, como a Baía da Babitonga, são mais suscetíveis à introduções de espécies exóticas que áreas da plataforma (Wasson et al., 2005). A presença de dois portos com grande fluxo de navios, em Itapoá e São Francisco do Sul, tem um papel preponderante e medidas de gestão como a raspagem periódica de organismos incrustantes dos cascos bem como a liberação da água de lastro em regiões oceânicas (onde organismos costeiros / estuarinos teriam menos chances de sobreviver), pode contribuir para evitar novas introduções. Ainda assim, há uma recente preocupação com invasões em áreas da plataforma adjacente, inclusive de espécies incrustadas em plataformas de petróleo (p. ex. gênero Tubastraea; Creed et al., 2017) ou em substratos flutuantes (Bumbeer \& Rocha, 2012).

Potenciais impactos ambientais dessas espécies exóticas estão principalmente relacionados à pressão de predação e/ou competição no caso dos estágios planctônicos (ver Nogueira Júnior \& Costa, 2019) e competição por espaço e/ou sobreposição da comunidade nativa no caso dos bênticos. Introduções em comunidades estabelecidas podem levar à substituição 
de espécies nativas por exóticas, com diminuição da abundância da primeira e eventual extinção local, levando a possíveis alterações na estrutura das comunidades e teia alimentar e, consequentemente, no fluxo energético (Molnar et al., 2008; Creed et al., 2017), porém são efeitos ainda não avaliados na Babitonga. Uma preocupação recente no litoral brasileiro, e que deve ser considerado para o $\mathrm{EB}$, é a rápida e crescente distribuição do coral invasor $\mathrm{Tu}$ bastraea spp., gênero com espécies com alto poder competitivo e de domínio da comunidade nativa e que já foi registrada no litoral catarinense (Ilha Galés, arquipélago Arvoredo e plataformas de óleo; Creed et al., 2017). Uma das principais medidas para minimizar o estabelecimento de espécies invasoras é o conhecimento da fauna nativa e de quais espécies tendem a ser mais perigosas aos ecossistemas nativos (Molnar et al., 2008), o que salienta a importância de levantamentos faunísticos em ambientes pouco estudados, como o EB.

Exóticos ou não, espécies de cnidários, especialmente macromedusas, podem influenciar negativamente na economia regional, particularmente no setor pesqueiro e turístico. São frequentes como fauna acompanhante em redes de pesca (Schroeder et al., 2014; Branco et $a l, 2015)$, gerando maiores custos na manutenção de redes e embarcações, bem como podem reduzir ou afastar o pescado alvo por compartilharem, direta ou indiretamente, os mesmos recursos (Nagata et al., 2009). As principais implicações no turismo referem-se aos acidentes com espécies tóxicas, como Chiropsalmus quadrumanus, Tamoya haplonema e Physalia physalis, a primeira registrada no estuário e plataforma do EB e as outras duas somente na plataforma (Tabela 1), problema também relatado por pescadores (Nagata et al., 2009). Portanto, conhecer padrões sazonais, amplamente desconhecidos para a maioria das espécies de macromedusas do EB, exceto $P$. punctata (Haddad \& Nogueira Júnior, 2006), pode auxiliar na gestão pesqueira, como o desenvolvimento de técnicas que diminuam a captura de fauna acompanhante e/ou definição de períodos e regiões menos propícias ao aparecimento de macromedusas, por exemplo, além da pre- caução de acidentes com espécies tóxicas.

\section{Conclusão}

Embora os estudos sobre cnidários no Ecossistema Babitonga sejam recentes e poucos, razoável conhecimento sobre a diversidade de Medusozoa foi gerado na região estuarina nesta última década. No entanto, provavelmente a riqueza de espécies aumentará consideravelmente com futuras investigações em costões rochosos e aumento do esforço amostral na região costeira e também estuarina no caso dos Anthozoa. Variações temporais de curta escala (e.g. ciclo de marés) bem como de médio e longo prazo não foram contempladas. A influência na economia e na comunidade marinha do EB também carece de informação, e trabalhos de perspectiva biológica e ecológica do grupo ajudarão a compreender a dinâmica e flutuações desses organismos e, consequentemente, de recursos pesqueiros locais e da prevenção de acidentes. Salienta-se a importância e intensificação do monitoramento da biodiversidade local considerando que o EB está susceptível a introduções de espécies exóticas (oito registros até o momento), inclusive de Tubastraea spp., espécies com elevado potencial de modificação da comunidade nativa e já registradas no litoral catarinense.

\section{Agradecimentos}

Os autores agradecem à Equipe Babitonga Ativa pela iniciativa e organização do projeto e auxílio nas buscas bibliográficas. ACC agradece à Coordenação de Aperfeiçoamento de Pessoal de Nível Superior (CAPES) pela bolsa de doutorado.

\section{Referências Bibliográficas}

ALTVATER, L. \& COUTINHO, R. 2015. Colonisation, competitive ability and influence of Stragulum bicolor van Ofwegen and Haddad, 2011 (Cnidaria, Anthozoa) on the fouling community in Paranaguá Bay, Southern Brazil. J. Exp. Mar. Biol. Eco., 462: 55-61.

BARDI, J. \& MARQUES, A. C. 2009. The invasive 
hydromedusae Blackfordia virginica Mayer, 1910 (Cnidaria: Blackfordiidae) in southern Brazil, with comments on taxonomy and distribution of the genus Blackfordia. Zootaxa, 2198: 41-50.

BARDI, J. 2011. Comunidades de hidrozoários (Cnidaria) estuarinos do sudeste e sul do Brasil. São Paulo. 188p. (Tese de doutorado. Instituto de Biociências, USP).

BOUILLON, J, MEDEL, M. D., PAGÈS, F., GILI, J. M., BOERO, F. \& GRAVILI, C. 2004. Fauna of the Mediterranean Hydrozoa. Sci. Mar., 68 (2): 1-454.

BOUZON, J. L., BRANDINI, F. P. \& ROCHA, R. M. 2012. Biodiversity of Sessile Fauna on Rocky Shores of Coastal Islands in Santa Catarina, Southern Brazil. Mar. Sci., 2: 39-47.

BRANCO, J. O., FREITAS JÚNIOR, F. \& CHRISTOFFERSEN, M. L. 2015 Bycatch fauna of seabob shrimp trawl fisheries from Santa Catarina State, southern Brazil. Biota Neotropica., 15: 1-14.

BRÜNING, A. L. B. 2008. Distribuição e ocorrência de Cnidaria Medusozoa no verão e inverno, na plataforma continental interna norte de Santa Catarina e sul do Paraná, Brasil. São Francisco do Sul. 25p. (Trabalho de conclusão de curso. Departamento de Ciências Biológicas, UNIVILLE).

BUMBEER, J. A. \& ROCHA, R. M. 2012. Detection of introduced sessile species on the near shore continental shelf in southern Brazil. Zoologia, 29: 126-134.

BUMBEER, J. A. \& ROCHA, R. M. 2016. Invading the natural marine substrates: a case study with invertebrates in South Brazil. Zoologia, 33: $1-7$.

CABRAL, A. C. 2013. Hidrozoários bênticos, em substrato artificial, como indicadores de condições ambientais na Baía da Babitonga, Santa Catarina. Curitiba. 114p. (Dissertação de mestrado. Setor de Ciências Biológicas, UFPR).

CANGUSSU, L. C., AltVATER, L., HADDAD, M. A., CABRAL, A. C., HEYSE, H. L. \& ROCHA, R. M. 2010. Substrate type as a selective tool against colonization by non-native sessile invertebrates. Braz. J. Oceanogr., 58: 219-
231.

CONCEPCIÓN, G. T., KAHNG, S. E., CREPEAU, M. W., FRANKLIN, E. C., COLES, S. L. \& TOONEN, R. J. 2010. Resolving natural ranges and marine invasions in a globally distributed octocoral (genus Carijoa). Mar. Ecol.Prog. Ser., 401: 113-127.

CORNELIUS, P. F. S. 1992. Medusa loss in leptolid Hydrozoa (Cnidaria), hydroid rafting, and abbreviated life-cycles among their remoteisland faunae: an interim review. Sci. Mar., 56: 245-261.

CREED, J. C., FENNER, D., SAMMARCO, P., CAIRNS, S., CAPEL, K., JUNQUEIRA, A. O. R., CRUZ, I., MIRANDA, R. J., CARLOSJÚNIOR, L., MANTELATTO, M. C. \& OIGMAN-PCZCZOL, S. 2017. The invasion of the azooxanthellate coral Tubastraea (Scleractinia: Dendrophylliidae) throughout the world: history, pathways and vectors. Biol. Invasions., 19: 283-305.

CREMER, M. J., MORALES, P.R.D. \& OLIVEIRA, T.M.N. 2006. Diagnóstico Ambiental da baía da Babitonga. Joinville, Editora da UNIVILLE. 256p.

DALY M., BRUGLER, M. R., CARTWRIGHT, P., COLLINS, A. G., DAWSON, M. N., FAUTIN, D. G., FRANCE, S. C., MCFADDEN, C. S., OPRESKO, D. M., RODRIGUEZ, E.; ROMANO, S. L. \& STAKE, J. L. 2007. The phylum Cnidaria: A review of phylogenetic patterns and diversity 300 years after Linnaeus. Zootaxa, 1668: 127-182.

GERHARDINGER, L.C., HERBST, D.F., CARVALHO, F.G., FREITAS, R.R., VILA-NOVA, D.; CUNHA, S., CREMER, M.J., PFUETZENREUTER, A., HAAK, L. 2017. Diagnóstico Socioambiental do Ecossistema Babitonga. $2^{\mathrm{a}}$ edição. Joinville, Editora da UNIVILLE. 271p.

GERHARDINGER, L.C., ZANK, S., CARVALHO, F.G., HERBST, D.S., CUNHA, S. \& CREMER, M.J. 2018. Governabilidade estrutural do subsistema natural do Ecossistema Babitonga (Santa Catarina: Brasil). Revista CEPSUL Biodiversidade \& Conservação Marinha, 7: eb2018001

GIBBONS, M. J., JANSON, L. A., ISMAIL, A. \& SAMAAI, T. 2010. Life cycle strategy, species 
richness and distribution in marine Hydrozoa (Cnidaria: Medusozoa). J. Biogeogr., 37: 441448.

GRAVILI, C. 2017. Alien jellyfish in expansion: the contribution of taxonomy to ecology. In: MARIOTTINI, G.L. (Org.). Jellyfish: ecology, distribution patterns and human interactions. Nova Publishers, New York, chap. 2: 27-49.

HADDAD, M. A. \& NOGUEIRA-JÚNIOR, M. 2006. Reappearance and seasonality of Phyllorhiza punctata von Lendenfeld (Cnidaria, Scyphozoa, Rhizostomeae) medusae in southern Brazil. Rev. Bras. Zool., 23: 824-831.

HADDAD, M. A. \& MARQUES, A. C. 2009. Cnidaria. In: ROCHA, R. M. \& BOEGER, W. A. P. (Org.). Estado da Arte e Perspectivas para a Zoologia no Brasil. Editora UFPR, Curitiba, cap. 3: 29-48.

HADDAD, M. A., BETTIM, A. L. \& MIGLIETTA, M. P. 2014. Podocoryna loyola, n. sp. (Hydrozoa, Hydractiniidae): a probably introduced species on artificial substrate from southern Brazil. Zootaxa, 3796: 494-506.

KAHNG, S. E. \& GRIGG, R. W. 2005. Impact of an alien octocoral, Carijoa riisei, on black corals in Hawaii. Coral Reefs, 24: 556-562.

MARQUES, A. C., MORANDINI, A. C. \& MIGOTTO, A. E. 2003. Synopsis of knowledge on Cnidaria Medusozoa from Brazil. Biota Neotropica, 3: 1-18.

MARQUES, A. C., KLÔH, A. S., MIGOTTO, A. E., CABRAL, A. C., RIGO, A. P. R., BETTIM, A. L., RAZZOLINI, E. L., CASCON, H. M., BARDI, J., KREMER, L. P., VIEIRA, L. M., BEZERRA, L. E. A., HADDAD, M. A., OLIVEIRA -FILHO, R. R., GUTIERRE, S. M. M., MIRANDA, T. P., FRANKLIN-JÚNIOR, W. \& ROCHA, R. M. 2013. Rapid assessment survey for exotic benthic species in the São Sebastião Channel, Brazil. Lat. Am. J. Aquat. Res., 41: 265-285.

MENON, D.M. 2006. Distribuição espacial dos hidróides (Cnidaria, Hydrozoa) do costão rochoso Pedra do Meio, Itapoá, Santa Catarina. Curitiba. 27p. (Monografia. Setor de Ciências Biológicas, UFPR).MOLNAR, J. L., GAMBOA, R. L., REVENGA, C. \& SPALDING, M. D. 2008. Assessing the global threat of inva- sive species to marine biodiversity. Front. Ecol. Environ., 6: 485-492.

MOLNAR, J.L., GAMBOA, R.L., REVENGA, C. \& SPALDING, M.D., 2008. Assessing the global threat of invasive species to marine biodiversity. Frontiers in Ecology and the Environment 6(9): 485 - 492. DOI: $10.1890 / 070064$

NAGATA, R. M., HADDAD, M. A. \& NOGUEIRAJÚNIOR, M. 2009. The nuisance of medusae (Cnidaria, Medusozoa) to shrimp trawls in central part of southern Brazilian Bight, from the perspective of artisanal fishermen. Panam. J. Aquat. Sci., 4: 312-325.

NOGUEIRA-JÚNIOR, M., ROBERT, M. C. \& HADDAD, M. A., 2006a. Calliactis tricolor (Anthozoa, Acontiaria) epibionte em Brachyura (Crustacea, Decapoda) no litoral sul do Paraná e Norte de Santa Catarina. Acta Biol. Par., 35: 233-248.

NOGUEIRA-JÚNIOR, M., ROBERT, M. C. \& HADDAD, M. A. 2006b.Ocorrência massiva de Aurelia sp. no sul do Brasil. Uma espécie nova?. In: XXVI Congresso Brasileiro de Zoologia, Londrina.

NOGUEIRA-JÚNIOR, M. \& SOUZACONCEIÇÃO, J. M. 2010. Seasonal distribution and size class composition of the scyphomedusae Chrysaora lactea (Semaeostomeae) and Lychnorhiza lucerna (Rhizostomeae) in a subtropical estuary in Southern Brazil. (Resumo de congresso). In Third International Jellyfish Blooms Symposium, Argentina.

NOGUEIRA-JÚNIOR, M. 2012. Gelatinous zooplankton fauna (Cnidaria, Ctenophora and Thaliacea) from Baía da Babitonga (southern Brazil). Zootaxa, 3398: 1-21.

NOGUEIRA-JÚNIOR, M., RODRIGUEZ, C. S., MIANZAN, H., HADDAD, M. A. \& GENZANO, G. N. 2013. Description of a new hydromedusa from the southwestern Atlantic Ocean, Bougainvillia pagesi sp. nov. (Cnidaria, Hydrozoa, Anthoathecata). Mar. Ecol., 34: 113-122.

NOGUEIRA-JÚNIOR, M., PUKANSKI, L. E. M. \& SOUZA-CONCEIÇÃO, J. M. 2015. Mesh size effects on assessments of planktonic hydrozoan abundance and assemblage structure. J. 
Marine. Syst., 144: 117-126.

NOGUEIRA-JÚNIOR, M. \& OLIVEIRA, V.M. 2017. Strategies of plankton occupation by polychaete assemblages in a subtropical estuary (south Brazil). J. Mar. Biol. Assoc. U.K., 97(8): 1651-1661 https://doi.org/10.1017/ $\underline{\text { So025315416001120 }}$

NOGUEIRA-JÚNIOR, M. \& BRANDINI, F.P. 2018. Community structure and spatialtemporal dynamics of the zooplankton in the South Brazilian Bight - a review. In: HOFFMEYER, M.S., SABATINI, M.E., BRANDINI, F.P., CALLIARI, D. \& SANTINELLI, N. (Org.) Plankton ecology of the Southwestern Atlantic - from the subtropical to the subantarctic realm. Springer, cahpter 10, pp.149-170

NOGUEIRA JÚNIOR, M. \& COSTA, M.D.P. 2019. Zooplâncton da Baía da Babitonga e plataforma continental adjacente: diagnóstico e revisão bibliográfica. Revista CEPSUL - Biodiversidade \& Conservação Marinha, 8: eb2019001

OLIVEIRA, A. F. S. 2014. Aspectos biológicos e ecológicos de zoantídeos zooxantelados em costões rochosos do sul e sudeste do Brasil. Florianópolis. 8op. (Dissertação de mestrado. Centro de Ciências Biológicas, UFSC).

OLIVEIRA, O. M. P., MIRANDA, T. P., ARAUJO, E. M., AYÓN, P., CEDEÑO-POSSO, C. M., CEPEDA-MERCADO, A. A., CÓRDOVA, P., CUNHA, A. F., GENZANO, G. N., HADDAD, M. A., MIANZAN, H. W., MIGOTTO, A. E., MIRANDA, L. S., MORANDINI, A. C., NAGATA, R. M., NASCIMENTO, K. B., NOGUEIRA -JÚNIOR, M., PALMA, S., QUIÑONES, J., RODRIGUEZ, C. S., SCARABINO, F., SCHIARITI, A., STAMPAR, S. N., TRONOLONE, V. B., \& MARQUES, A. C. 2016. Census of Cnidaria (Medusozoa) and Ctenophora from South American marine waters. Zootaxa, 4194: 1-256.

PAGLIOSA, P. R. 2006. Distribuição da macrofauna bêntica do entremarés ao sublitoral em uma praia estuarina da Baía da Babitonga, Sul do Brasil. Revista Biotemas, 19: 25-33.

PUKANSKI, L. E. M., HADDAD, M. A., NOGUEIRA-JÚNIOR, M. \& SOUZA-CONCEIÇÃO, J. M. 2010. Distribuição Espaço-Temporal das hidromedusas: Liriope tetraphylla (Trachymedusae) e Blackfordia virginica
(Leptothecata) na Baía da Babitonga, Santa Catarina. (Resumo de Congresso). In III Congresso Brasileiro de Oceanografia, Rio Grande.

PUKANSKI, L. E. M. 2011. Dinâmica populacional das hidromedusas Liriope tetraphylla (Trachymedusae) e Blackfordia virginica (Leptothecata) na Baía da Babitonga - litoral norte de Santa Catarina, Brasil. Curitiba. 39p. (Dissertação de mestrado. Departamento de Zoologia, UFPR).

SCHROEDER, R., BRANCO, J. O., FREITAS F. \& RESGALLA C. 2014. Preliminary assessment of the jellyfish bycatch captured off southern and southeastern Brazil. Lat. Am. J. Aquat. Res., 42: 289-300.

VAN OFWEGEN, L. P. \& HADDAD, M. A. 2011. A probably invasive new genus and new species of soft coral (Octocorallia: Alcyonacea: Clavulariidae) from Brazil. Zootaxa 3107: 38-46.

VIDOLIN, D. 2007. Tolerância à variação de parâmetros ambientais e sua influência sobre a distribuição espacial de anêmonas-do-mar na zona entremarés de um costão rochoso no sul do Brasil. Curitiba. 27p. (Tese de doutorado. Setor de Ciências Biológicas, UFPR).

WASSON, K., FENN, K. \& PEARSE, J.S. 2005. Habitat differences in marine invasions of central California. Biological Invasions 7: 935 $-948$.

YOSHIMURA, C. Y. 1997. Estudo da distribuição vertical e variação sazonal de espécies da zona entremarés em costão rochoso da praia de Itapema do Norte, Santa Catarina, Brasil. Curitiba. 29p. (Trabalho de Conclusão de Curso. Setor de Ciências Biológicas, UFPR). 